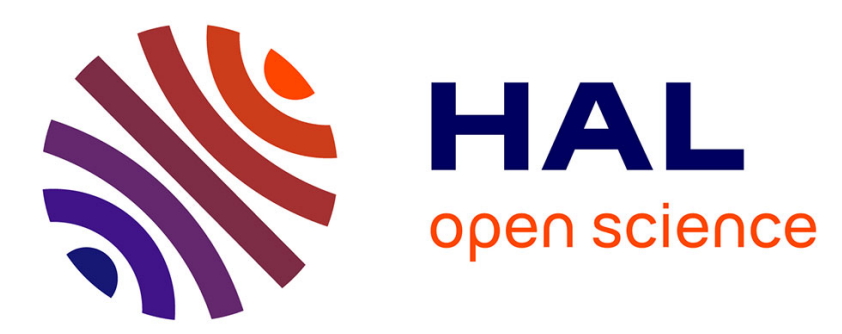

\title{
Réalisation d'un spectromètre de coïncidences à sept compteurs NaI
}

\author{
S. Gorodetzky, F.A. Beck, P. Engelstein, T. Byrski, A. Knipper
}

\section{To cite this version:}

S. Gorodetzky, F.A. Beck, P. Engelstein, T. Byrski, A. Knipper. Réalisation d'un spectromètre de coïncidences à sept compteurs NaI. Revue de Physique Appliquée, 1969, 4 (2), pp.103-104. 10.1051/rphysap:0196900402010300 . jpa-00243164

\section{HAL Id: jpa-00243164 https://hal.science/jpa-00243164}

Submitted on 1 Jan 1969

HAL is a multi-disciplinary open access archive for the deposit and dissemination of scientific research documents, whether they are published or not. The documents may come from teaching and research institutions in France or abroad, or from public or private research centers.
L'archive ouverte pluridisciplinaire HAL, est destinée au dépôt et à la diffusion de documents scientifiques de niveau recherche, publiés ou non, émanant des établissements d'enseignement et de recherche français ou étrangers, des laboratoires publics ou privés. 


\title{
RÉALISATION D’UN SPECTROMÈTRE DE GOÏNGIDENGES A SEPT GOMPTEURS NaI
}

\author{
S. GORODETZKY, F. A. BECK, P. ENGELSTEIN, T. BYRSKI et A. KNIPPER, \\ Centre de Recherches Nucléaires, 67-Strasbourg.
}

\begin{abstract}
Résumé. - Un multicompteur comprenant sept détecteurs $\mathrm{NaI}(\mathrm{Tl}) 3^{\prime \prime} \times 3^{\prime \prime}$ a été construit. Il permet de mesurer simultanément les valeurs correspondant à 16 angles de la fonction de corrélation angulaire $W\left(\theta_{1}, \theta_{2}, \varphi\right)$ ou les sept géométries définies par Litherland et Ferguson. Cet ensemble a été expérimenté avec succès dans le cas de corrélations angulaires bien connues aussi bien en désintégrations radioactives qu'en réactions nucléaires.
\end{abstract}

Abstract. - A seven detector $\mathrm{NaI}(\mathrm{Tl}) 3^{\prime \prime} \times 3^{\prime \prime}$ multicounter has been built. It allows the simultaneous measurements of the corresponding sixteen values of the angular correlation function $W\left(\theta_{1}, \theta_{2}, \varphi\right)$ or the Litherland and Ferguson seven geometries. This apparatus has been successfully tested in the case of well known angular correlations in radioactive decays as well as in nuclear reactions.

I. Introduction. - Depuis que Litherland et Ferguson $[1,2]$ ont développé leur formalisme des corrélations angulaires avec niveau initial orienté, l'étude de celles-ci a connu un grand essor. En effet, ce formalisme permet de décrire la fonction de corrélation, qui dépend de deux angles polaires et d'un angle azimutal, à partir des populations $P(m)$ du niveau aligné. Litherland et Ferguson ont défini sept " géométries 》 en fixant deux angles et en faisant varier le troisième. Cette méthode a souvent été utilisée. Les sept fonctions à mesurer ont des expressions simples (développables en polynômes de Legendre directs, de coefficients $\mathrm{A}_{2}$ et $\mathrm{A}_{4}$ ) et les corrections de normalisation sont aisées. Cependant, les temps de mesure sont longs et la détermination des coefficients de mélange à partir des coefficients $A_{2}$ et $A_{4}$ est délicate, surtout dans le cas d'un double mélange. Une amélioration considérable de précision, dans l'expérience et dans l'analyse, peut être obtenue en définissant la fonction de corrélation angulaire par la mesure simultanée des coïncidences entre plusieurs compteurs.

II. Description de l'appareillage. - II.1. DesGRIPTION GÉNÉRALE. - Les sept compteurs $\mathrm{NaI}(\mathrm{Tl})$ $3^{\prime \prime} \times 3^{\prime \prime}$ ont été disposés dans deux plans orthogonaux suivant les angles représentés sur la figure 1. Une boîte de cible sphérique montée à demeure permet la mesure de corrélations angulaires $\gamma-\gamma$ en réactions nucléaires (niveau initial aligné) et en désintégrations radioactives (une source remplaçant l'impact du faisceau sur la cible). Un système de huit diaphragmes de $2,3 \mathrm{~mm}$, séparés deux à deux par six centimètres de plomb, assure une bonne reproductibilité du point d'impact du faisceau sur la cible. L'ensemble rigide compteurs + boîte de cible est mobile dans les trois directions

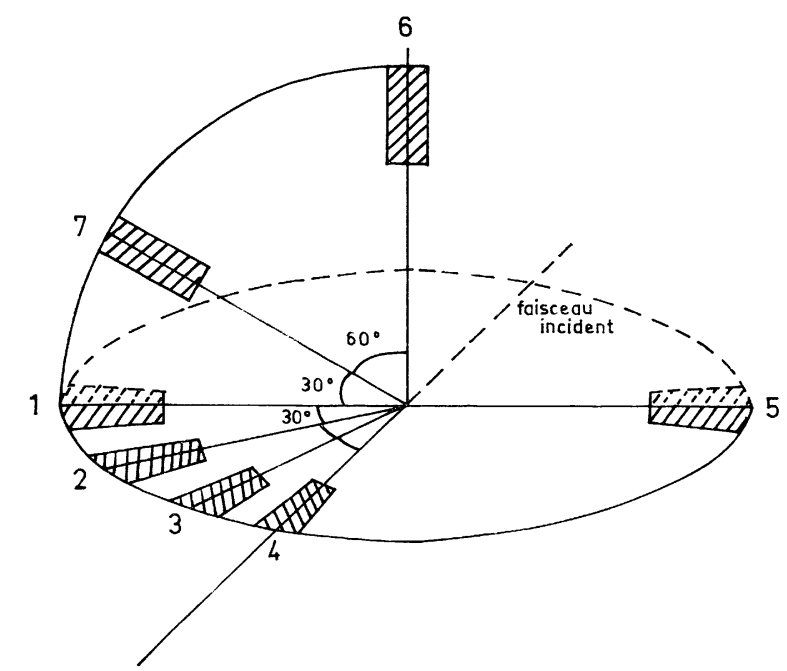

FIG. 1. - Disposition dans l'espace des sept compteurs du multicompteur.

de l'espace. Le multicompteur permet la mesure de vingt et une valeurs $(n(n-1) / 2)$ de la fonction de corrélations angulaires qui ne sont pas toutes linéairement indépendantes. Nous en avons sélectionné seize qui permettent à la fois de retrouver les sept géométries et d'analyser la corrélation angulaire en valeurs d'angles. Un commutateur permet de passer des mesures de corrélations angulaires à une mesure de distributions angulaires à sept compteurs.

iI.2. Élegtronioue associée. - Le schéma de l'électronique associée au multicompteur peut être compris à partir d'un système simple à deux compteurs 


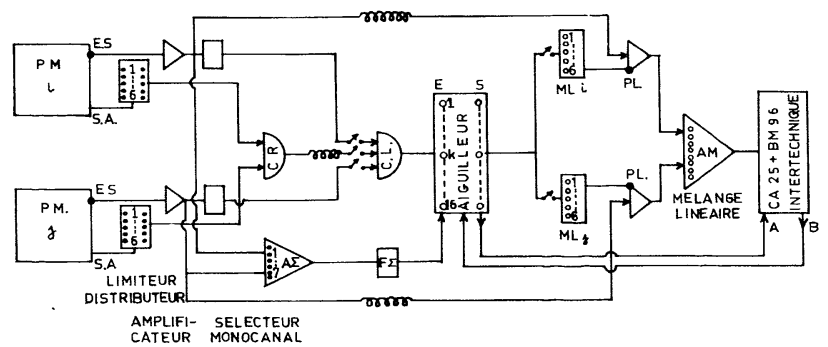

FIG. 2. - Schéma synoptique de base (deux compteurs) du circuit électronique associé au multicompteur.

(fig. 2). La technique de coïncidence somme [3] est particulièrement bien adaptée aux mesures de corrélations angulaires, elle permet en outre l'analyse simultanée des géométries paires et impaires (en fait on mesure trente-deux valeurs au lieu de seize). On améliore les performances de la méthode en imposant sur les voies linéaires de chaque compteur un discriminateur différentiel et une porte linéaire. Celle-ci est déclenchée par une impulsion qui comprend les informations de temps et d'énergie. Cette impulsion provient d'un aiguilleur par l'intermédiaire d'un circuit mélangeur chargé de distribuer les permissions d'analyse aux différents compteurs. L'utilisation d'un aiguilleur à seize voies permet l'extension de la mesure simultanée de deux à trente-deux valeurs de la fonction de corrélation angulaire avec le même convertisseur C.A.D. 25 grâce à une partition d'un bloc mémoire BM 96 Inter- technique en seize groupes de 256 canaux. De plus, pour minimiser les fluctuations instrumentales dans le temps, on intercale sur chaque voie linéaire un stabilisateur analogique digital utilisant la seconde partie du C.A.D. 25; chacun des stabilisateurs est déclenché par une sortie correspondante de l'aiguilleur. Tous les circuits électroniques, sauf les correcteurs de dérive, ont été réalisés en laboratoire et longuement éprouvés.

III. Normalisation de l'appareillage. - Le multicompteur a été expérimenté ‘en source radioactive (dans ce cas, il n'y a que sept angles indépendants) et en réaction nucléaire. Ainsi, nous avons mesuré avec précision les corrélations angulaires dans les désintégrations radioactives de ${ }^{60} \mathrm{Co},{ }^{207} \mathrm{Bi}$ et ${ }^{106} \mathrm{Ru}$. En réaction nucléaire, les corrélations angulaires entre des rayons $\gamma$ impliquant un niveau intermédiaire de moment angulaire 0 et $1 / 2$ doivent être isotropes dans certaines géométries, et égales à la distribution angulaire du rayon $\gamma$ alimentant le niveau intermédiaire dans d'autres. Nous avons ainsi remesuré, avec une bonne précision, les corrélations angulaires entre les rayons $\gamma$ de 580 et $400 \mathrm{keV}$ obtenus dans la réaction ${ }^{25} \mathrm{Mg}\left(\mathrm{p}, \mathrm{p}^{\prime}\right)$ et entre des rayons $\gamma$ de 1,63 et $2,31 \mathrm{MeV}$ obtenus dans la réaction ${ }^{12} \mathrm{C}\left({ }^{3} \mathrm{He}, \mathrm{p}\right){ }^{14} \mathrm{~N}$. Signalons enfin que cet ensemble peut également être utilisé dans l'étude de corrélations angulaires particule- $\gamma$ et qu'il l'a effectivement été dans la mesure de corrélations angulaires $\mathrm{p}-\gamma$ pour la détermination du mécanisme de la réaction ${ }^{11} \mathrm{~B}(\mathrm{~d}, \mathrm{p})^{12} \mathrm{~B}$.

\section{BIBLIOGRAPHIE}

[1] LiTheriand (A. E.) et Ferguson (A. J.), Can. J. Phys., 1961, 39, 788.

[2] FERGUSON (A. J.), Angular correlation methods in gamma-ray spectroscopy, North-Holland, Amsterdam, 1965.

[3] Hoogendoom (A. M.), Nucl. Instr., 1958, 3, 57. 